\title{
Tác động của quản trị công lên đầu tư công ở các quốc gia đang phát triển: Bằng chứng thực nghiệm
}

\section{The effect of governance environment on public investment in developing countries: Empirical evidence}

\author{
Nguyễn Văn Bổn ${ }^{1 *}$, Trần Thị Mỹ Phước ${ }^{2}$ \\ ${ }^{1}$ Khoa Tài chính Ngân hàng, Trường Đại học Tài chính Marketing, Việt Nam \\ ${ }^{2}$ Khoa Tài chính Kế toán, Trường Đại học Sài Gòn, Việt Nam \\ *Tác giả liên hệ, Email: boninguyen@gmail.com
}

THÔNG TIN

TÓM TẮT

DOI: $10.46223 / \mathrm{HCMCOUJS.}$ econ.vi.16.1.558.2021

Ngày nhận: 24/06/2020

Ngày nhận lại: 06/07/2020

Duyệt đăng: 07/07/2020

Tù khóa:

đầu tư công, môi trường quản trị công, phương pháp GMM hệ thống, các quốc gia đang phát triển

Keywords:

public investment, governance environment, system GMM Arellano-Bond estimator, developing countries.
Môi trường thể chế đóng vai trò quan trọng trong quá trình phát triển kinh tế ở hầu hết các quốc gia trên thế giới. Sự khác biệt trong môi trường thể chế đưa đến sự khác biệt trong các hoạt động kinh tế giữa các quốc gia. Bài viết này đánh giá tác động của môi trường quản trị công (thể chế) lên đầu tư công ở 72 quốc gia đang phát triển trong giai đoạn 2002 - 2018 bằng phương pháp ước lượng $\mathrm{GMM}$ Arellano-Bond hệ thống. Phát hiện cho thấy môi trường quản trị công làm giảm đầu tư công ở các quốc gia này. Ngoài ra, tăng trưởng kinh tế thúc đẩy trong khi lạm phát và cơ sở hạ tầng làm giảm đầu tư công. Các phát hiện trong nghiên cứu này đề xuất một số hàm ý chính sách quan trọng cho chính phủ ở các nước đang phát triển, đặc biệt Việt Nam, trong quá trình cải thiện môi trường quản trị công để gia tăng thúc đẩy đầu tư công, phục vụ cho phát triển kinh tế.

ABSTRACT
The institutional environment plays a critical role in the process
of economic development in most countries worldwide. The
difference in the institutional environment leads to a difference in
the level of development between countries. The paper empirically
investigates the effect of the governance environment on public
investment for 72 developing countries during the period of 2002 -
2018 using the system GMM Arellano-Bond estimators. The
findings indicated that the governance environment hinders public
investment in these countries. Besides, economic growth stimulates
while inflation and infrastructure reduce public investment. These
findings suggest some important policy implications for
governments in developing countries, especially Vietnam, in
reforming the governance environment to promote public
investment for economic development.

\section{Giới thiệu}

Mối quan hệ giữa môi trường thể chế và đầu tư công là một trong các chủ đề quan trọng giữa các nhà kinh tế học. Bắt đầu từ công trình nghiên cứu của Tanzi và Davoodi (1998), một dòng 
nghiên cứu nhỏ đã và đang nghiên cứu mối quan hệ này nhằm chứng minh cho tác động thúc đẩy hoặc cản trở đầu tư công của môi trường thể chế. Trong nghiên cứu, có một sự nhất trí cao giữa các nhà kinh tế học và các nhà chính trị là vốn công đóng vai trò quan trọng trong phát triển kinh tế bởi vì nó góp phần vào việc cải thiện cơ sở hạ tầng và gia tăng vốn con người (Haughwout, 2002). Cơ sở hạ tầng có thể làm giảm chi phí cố định, thu hút các doanh nghiệp và các yếu tố sản xuất, và qua đó nâng cao sản xuất. Trong khi đó, môi trường thể chế có thể được xem là nguyên nhân nền tảng của phát triển dài hạn ở các quốc gia trên thế giới. North (1990) định nghĩa "thể chế là những luật lệ của cuộc chơi trong xã hội”. Cụ thể hơn thì thể chế là những ràng buộc do con người tạo ra để để hiệu chỉnh và định hình các tương tác của mình. Dựa trên cách tiếp cận vĩ mô thì lý thuyết thể chế của North (1990) lập luận trong trường hợp thị trường bị chi phối bởi các yếu tố chính trị và kinh tế là hiệu quả, nghĩa là không có chi phí giao dịch, thì mọi lựa chọn sẽ luôn đạt hiệu quả. Ở một khía cạnh khác, Acemoglu, Johnson, và Robinson (2005) nhấn mạnh sự khác biệt trong môi trường thể chế đưa đến sự khác biệt trong các hoạt động kinh tế giữa các quốc gia. Đặc biệt, các quốc gia đang phát triển phần lớn đều sử dụng chính sách tài khóa, cụ thể là chi tiêu đầu tư công để thúc đẩy tăng trưởng kinh tế, tạo nhiều việc làm và ổn định xã hội. Điều này đưa đến câu hỏi nghiên cứu "Môi trường quản trị công ở các nước đang phát triển liệu có tạo điều kiện thuận lợi cho việc thúc đẩy đầu tư công hay không?”.

Liên quan đến chủ đề nghiên cứu, chúng tôi có một số lập luận để lý giải cho sự tác động của môi trường quản trị công lên đầu tư công như sau. Dưới môi trường quản trị công kém, các dự án đầu tư công không được kiểm soát chặt chẽ và giám sát công khai. Hầu hết các dự án đầu tư công được thực thi mà không có sự tham gia của người dân, vì thế tham nhũng xuất hiện. Tham nhũng có thể làm sai lệch toàn bộ quá trình ra quyết định gắn với ngân sách đầu tư của chính phủ. Các hiện tượng giả tạo như "voi trắng" và "lâu đài trên cát" trong các dự án công được tạo ra (Tanzi \& Davoodi, 1998). Các viên chức chính phủ có thể trình ra một báo cáo sai lệch về quá trình mua sắm chất lượng cao chi phí cao, nhưng cung cấp các sản phẩm chất lượng thấp giá thấp (Haque \& Kneller, 2015). Vì thế, tham nhũng có thể làm tăng lượng vốn đầu tư cho các dự án công, nhưng làm giảm tỷ suất sinh lời trên các nguồn vốn đó. Đặc biệt, do thiếu giám sát công khai và kiểm soát minh bạch, việc thiết kế, hình thành và thực thi các chính sách liên quan đến đầu tư công và các dự án đầu tư công bị tác động bởi các nhóm lợi ích và các viên chức trục lợi. Nhiều dự án đầu tư công không gắn kết với lợi ích của người dân. Trong khi đó, việc cải thiện môi trường quản trị công sẽ dần loại trừ sự trục lợi của các viên chức chính phủ, lợi ích nhóm và các lỗ hổng luật pháp trong quá trình thiết kế, ban hành và thực thi các chính sách liên quan đến đầu tư công. Do vậy, việc cải thiện môi trường quản trị công sẽ làm giảm đầu tư công.

Xuất phát từ bối cảnh môi trường quản trị công có thể tác động có ý nghĩa lên đầu tư công ở các nước đang phát triển và trả lời cho câu hỏi nghiên cứu trên, chúng tôi đánh giá thực nghiệm mối quan hệ giữa môi trường quản trị công và đầu tư công cho 72 quốc gia đang phát triển trong khoảng thời gian 2002 - 2018 với các biến kiểm soát như tăng trưởng kinh tế, độ mở thương mại, lạm phát, và cơ sở hạ tầng bằng phương pháp ước lượng GMM Arellano-Bond hệ thống hai bước. Tính bền của nghiên cứu sẽ được kiểm tra bằng phương pháp ước lượng GMM Arellano-Bond hệ thống một bước. So với các nghiên cứu trước đây cùng chủ đề thì bài viết này thể hiện sự khác biệt (tính mới) dựa trên hai khía cạnh: (i) sử dụng 6 thành phần quản trị công của World Bank đại diện cho môi trường thể chế; và (ii) sử dụng phương pháp ước lượng GMM Arellano-Bond hệ thống hai bước cho việc ước lượng và phương pháp ước lượng GMM Arellano-Bond hệ thống một bước để kiểm tra tính bền.

Bài viết được cấu trúc như sau. Ngoài Phần 1 là giới thiệu thì Phần 2 là tổng quan các nghiên cứu trước, tập trung vào mối quan hệ giữa thể chế/quản trị công/tham nhũng và đầu tư công. Mô hình và dữ liệu nghiên cứu được trình bày ở Phần 3 với việc nhấn mạnh vào sự phù hợp 
của các phương pháp ước lượng GMM Arellano-Bond hệ thống. Phần 4 trình bày các kết quả ước lượng, bàn luận và kiểm tra tính bền. Phần cuối cùng kết luận và đề xuất một vài hàm ý quan trọng từ các phát hiện trong Phần 4.

\section{Tổng quan các nghiên cứu trước đây}

Tham nhũng làm móp méo toàn bộ quá trình ra quyết định gắn với các dự án đầu tư công; đặc biệt môi trường quản trị công càng yếu kém thì mức độ móp méo càng cao (Tanzi \& Davoodi, 1998). Bằng cách sử dụng phương pháp ước lượng OLS cho mẫu của 92 quốc gia trong giai đoạn 1982 - 1995, Tanzi và Davoodi (1998) chỉ ra tham nhũng làm tăng đầu tư công. Tương tự, mô hình tăng trưởng nội sinh với thông tin bất cân xứng giữa chính phủ và các quan chức được phát triển bởi Haque và Kneller (2015) để lý giải tại sao đầu tư công thất bại trong việc thúc đẩy tăng trưởng kinh tế ở các quốc gia nơi tham nhũng có tính địa phương. Để minh chứng quan điểm này, Haque và Kneller (2015) sử dụng phương pháp ước lượng 3SLS cho mẫu của 66 quốc gia từ 1970 đến 2000. Kết quả thực nghiệm cho thấy: (1) tham nhũng làm tăng đầu tư công; (2) tham nhũng làm giảm tỷ suất sinh lợi trên vốn đầu tư công và không hiệu quả trong việc thúc đẩy tăng trưởng kinh tế. Trong khi đó, Grigoli và Mills (2014) lập luận rằng tầng lớp tinh hoa ở các quốc gia với thể chế kém sử dụng đầu tư công như cỗ máy phục vụ cho lợi ích của họ. Grigoli và Mills (2014) sử dụng các phương pháp ước lượng GMM sai phân và hệ thống để kiểm tra thực nghiệm ảnh hưởng của chất lượng thể chế lên số lượng, sự thay đổi và chất lượng của đầu tư công cho dữ liệu bảng của 144 quốc gia trong giai đoạn 1984 - 2008. Các kết quả ước lượng cho thấy môi trường quản trị công có tác động âm lên đầu tư công, ủng hộ giả thuyết là chính phủ sử dụng đầu tư công như một phương tiện để trục lợi. Trái lại, Agyei (2017) cho thấy việc cải thiện môi trường quản trị công thúc đẩy vốn đầu tư công. Agyei (2017) đánh giá thực nghiệm ảnh hưởng của cấu trúc quản trị công lên đầu tư công cho dữ liệu bảng không cân bằng của 48 quốc gia vùng Hạ Sahara Châu Phi (SSA) trong thời gian 1990 - 2009 bằng cách sử dụng phương pháp ước lượng sai phân một bước. Các kết quả ước lượng đề nghị cấu trúc quản trị công làm gia tăng đầu tư công ở SSA.

Trái lại, De la Croix và Delavallade (2009) khẳng định rằng ảnh hưởng của tham nhũng lên đầu tư công không phải lúc nào cũng giống nhau. Tham nhũng có thể hạn chế khả năng của các quốc gia nghèo bắt kịp các quốc gia giàu có. Điều này có thể làm móp méo việc phân bổ chi tiêu công. Thực vậy, Delavallade (2006) đánh giá thực nghiệm tác động của tham nhũng lên thành phần chi tiêu công theo lĩnh vực bằng cách sử dụng phương pháp ước lượng 3SLS cho mẫu 64 quốc gia từ 1996 đến 2001. Các kết quả ước lượng chỉ ra tham nhũng làm giảm phần chi tiêu sử dụng cho giáo dục, y tế và bảo vệ xã hội và làm tăng phần chi tiêu dành cho dịch vụ công, xăng dầu, văn hóa, và quốc phòng. Tương tự như vậy, De la Croix và Delavallade (2009) sử dụng phương pháp ước lượng 2SLS cho mẫu 62 quốc gia trong thời gian từ 1996 đến 2004. Các phát hiện nhấn mạnh rằng tác động của hệ thống tư pháp kém lên cấu trúc của đầu tư công tùy vào trình độ phát triển của một quốc gia.

Không như các nghiên cứu trên, một số nghiên cứu khác sử dụng các đo lường về thể chế có tính khác biệt như các dạng ảnh hưởng của doanh nghiệp (Duchin \& Sosyura, 2012), thể chế và luật lệ phi chính thống (Xu \& Yao, 2015), và chế độ chính trị (Gwatipedza \& Janus, 2019). Duchin và Sosyura (2012) nghiên cứu mối quan hệ giữa các dạng ảnh hưởng chính trị của doanh nghiệp như sân nhà của các chính trị gia, vận động hành lang, đóng góp chiến dịch tranh cử lên đầu tư công. Bằng cách sử dụng các dữ liêu thu thập dựa trên các đơn xin cấp vốn của doanh nghiệp theo Chương trình TARP, nghiên cứu phát hiện đầu tư đến từ tài trợ của chính phủ dành cho các doanh nghiệp có ảnh hưởng chính trị có tính kém hiệu quả so với đầu tư đến từ tài trợ của chính phủ dành cho các doanh nghiệp không có ảnh hưởng chính trị. Trong khi đó, Xu và Yao (2015) lại đặt vấn đề liệu các thể chế, luật lệ và chuẩn mực phi chính thống được tạo dựng và giám 
sát bởi các nhóm xã hội có thúc đẩy đầu tư công ở địa phương hay không xét trong bối cảnh tính dân chủ thấp và tệ quan liệu cao? Để trả lời cho câu hỏi tương đối khó này, hai nhà nghiên cứu sử dụng bộ dữ liệu bảng của 220 ngôi làng ở Trung Quốc từ 1986 đến 2005 . Kết quả cho thấy các thể chế phi chính thống này thúc đẩy việc gia tăng đầu tư công ở địa phương. Mới nhất, Gwatipedza và Janus (2019) phân tích và đánh giá thực nghiệm sự khác biệt về hiệu quả của đầu tư công giữa các quốc gia độc tài và các quốc gia dân chủ. Kết quả cho thấy đầu tư của chính phủ ở các quốc gia độc tài thay đổi nhiều và kém hiệu quả hơn so với đầu tư của chính phủ ở các quốc gia dân chủ.

\section{Mô hình và dữ liệu nghiên cứu}

\subsection{Mô hình nghiên cứu}

Dựa trên nghiên cứu của Grigoli và Mills (2014), phương trình thực nghiệm được mở rộng như sau:

$$
G I N_{i t}=\beta_{0}+\beta_{1} G I N_{i t-1}+\beta_{2} G O V_{i t}+X_{i t} \beta^{\prime}+\eta_{i}+\xi_{i t}
$$

với $i$ và $t$ lần lượt ký hiệu cho quốc gia và thời gian. $G I N_{i t}$ là đầu tư công (\%GDP), GIN $N_{i t-1}$ đại diện cho vốn đầu tư công lúc ban đầu, và $G O V_{i t}$ là môi trường quản trị công (bao gồm 6 biến quản trị công, bao gồm kiểm soát tham nhũng, hiệu quả chính phủ, ổn định chính trị, chất lượng luật lệ, nhà nước pháp quyền, tiếng nói và giải trình). $X_{i t}$ là một tập các biến kiểm soát như tăng trưởng kinh tế (GDP bình quân đầu người thực), độ mở thương mại (tỷ lệ xuất nhập khẩu theo GDP), lạm phát, và cơ sở hạ tầng (số thuê bao điện thoại cố định trên 100 dân); $\eta_{i}$ là tác động cố định không quan sát được có đặc điểm quốc gia, bất biến theo thời gian và $\zeta_{i t}$ là đại lượng sai số; $\beta_{0}, \beta_{1}, \beta_{2}$, và $\beta$ ' là các hệ số ước lượng.

Có bốn vấn đề nghiêm trọng về kinh tế lượng từ việc ước lượng phương trình (1). Thứ nhất, một vài biến như tăng trưởng kinh tế, cơ sở hạ tầng, quản trị công có thể mang tính nội sinh. Các biến này có thể tương quan với đại lượng sai số $\eta_{i}$, và đưa đến hiện tượng nội sinh. Thứ hai, một vài đặc điểm không quan sát được, bất biến theo thời gian và có đặc tính quốc gia (tác động cố định) như địa lý và nhân khẩu học có thể tương quan với các biến độc lập. Các tác động cố định này hiện diện trong đại lượng sai số $\eta_{i}$. Thứ ba, sự hiện diện của biến phụ thuộc có độ trễ GIN $N_{i t-1}$ dẫn đến tự tương chuỗi quan cao. Cuối cùng, dữ liệu bảng có khoảng thời gian quan sát ngắn $(\mathrm{T}=$ 17) và số đơn vị bảng nhiều $(\mathrm{N}=72)$. Những vấn đề này có thể khiến ước lượng OLS không nhất quán và bị chệch. Mô hình các tác động cố định FEM và mô hình các tác động ngẫu nhiên REM không xử lí được hiện tượng nội sinh và tự tương quan chuỗi trong khi ước lượng PMG (Pool Mean Group) và ước lượng MG (Mean Group) cần một khoảng thời gian quan sát dài để ước lượng cả trong ngắn hạn lẫn dài hạn. Thêm vào đó, ước lượng IV-2SLS đòi hỏi một vài biến công cụ thích hợp nằm ngoài các biến độc lập trong mô hình. Vì thế, chúng tôi quyến định lựa chọn phương pháp ước lượng sai phân được đề xuất bởi Judson và Owen (1999).

Phương trình (1) sẽ được dùng để đánh giá mối quan hệ giữa môi trường quản trị công đầu tư công cho 72 quốc gia đang phát triển. Trong nghiên cứu này, chúng tôi sử dụng 6 biến quản trị công được xây dựng bởi dự án Worldwide Governance Indicators (Kaufmann, Kraay, \& Mastruzzi, 2011), để đại diện cho môi trường quản trị công. "Quản trị công là quá trình qua đó các đối tượng thuộc nhà nước và bên ngoài tương tác với nhau để thiết kế và thực thi các chính sách trong điều kiện các luật lệ chính thống và phi chính thống định hình nên quyền lực và cũng được hình thành bởi quyền lực" và "Quyền lực là khả năng của một nhóm người hay một nhóm các cá nhân có tác động lẫn nhau dựa trên các lợi ích của nhau và mang lại các thành quả nhất." (World Bank, 2017, p. 3). Sáu biến quản trị công được đo lường theo các điểm số của chỉ số quản trị công 
trong khoảng -2.5 đến 2.5 . Việc thiếu môi trường quản trị công tốt ở hầu hết các quốc gia đang phát triển dẫn đến các tác động tiêu cực lên triển vọng phát triển kinh tế; vì thế cải thiện môi trường quản trị công đóng vai trò quan trọng trong nghị trình phát triển chính ở các quốc gia này (Hope, 2009). Một môi trường quản trị công tốt đóng góp có ý nghĩa vào sự hình thành chính phủ kiến tạo với khả năng thực thi các chính sách phát triển.

Chúng tôi sử dụng các phương pháp ước lượng GMM Arellano và Bond (1991) lần đầu được đề xuất bởi Holtz-Eakin, Newey, và Rosen (1988). Để ước lượng phương trình (1), chúng tôi lấy sai phân bậc một để loại trừ các tác động cố định có đặc tính quốc gia $\left(\eta_{i}\right)$. Sau đó, các biến độc lập ở dạng sai phân bậc nhất được sử dụng như các biến được công cụ theo độ trễ của chúng dưới giả định rằng các sai số thay đổi theo thời gian trong các mô hình gốc không có tương quan chuỗi (Judson \& Owen, 1999). Chiến lược này là GMM sai phân, được biết là có khả năng xử lí các hiện tượng chệch do tác động đồng thời trong các ước lượng.

Phương trình (1) có thể được chuyển đổi thành phương trình dưới dạng sai phân bậc nhất như sau:

$$
\begin{gathered}
\operatorname{GIN}_{i t}-\operatorname{GIN}_{i t-1}=\beta_{1}\left(G I N_{i t-1}-G I N_{i t-2}\right)+\beta_{2}\left(G O V_{i t}-G O V_{i t-1}\right) \\
+\left(X_{i t}-X_{i t-1}\right) \beta^{\prime}+\left(\xi_{i t}-\xi_{i t-1}\right)
\end{gathered}
$$

Trong trường hợp các biến có tính dai dẳng, nghĩa là các giá trị quá khứ cho thấy khá ít thông tin về sự biến đổi trong tương lai, khiến cho các biến trễ trở thành các công cụ yếu ở dạng sai phân. Vì thế, Arellano và Bover (1995) đề nghị kết hợp phương trình (1) và phương trình (2) để hình thành một hệ gồm hai phương trình, một phương trình dạng sai phân được công cụ bởi các biến trê̂,, và một phương trình dạng gốc được công cụ bởi các biến trễ sai phân qua đó GMM được áp dụng. Đây là phương pháp ước lượng GMM hệ thống, một chiến lược có khả năng tăng tính hiệu quả thông qua việc giảm bớt hiện tượng chệch và giải quyết được các biến công cụ yếu ở GMM sai phân. Tính nhất quán của GMM hệ thống hoàn toàn dựa trên các giả định là các đại lượng sai số không tương quan nhau, các biến công cụ có hiệu lực, và các thay đổi trong các biến công cụ thêm vào không tương quan với các tác động cố định.

Các ước lượng GMM sai phân hai bước có tính hiệu quả hơn các ước lượng GMM sai phân một bước. Tuy nhiên, việc áp dụng các ước lượng GMM sai phân hai bước trong các mẫu dữ liệu nhỏ, chẳng hạn ở nghiên cứu này, có một vài vấn đề (Roodman, 2009). Những vấn đề này hình thành từ việc gia tăng theo cấp số nhân số lượng biến công cụ khi khoảng thời gian tăng lên. Điều này có thể khiến số lượng biến công cụ quá nhiều so với số lượng các quốc gia. Để tránh việc này, quy tắc ngón trỏ nên được áp dụng để duy trì số lượng biến công cụ nhỏ hơn hoặc bằng số lượng các đơn vị bảng (số quốc gia) (Roodman, 2009).

Tính hiệu lực của các biến công cụ trong phương pháp GMM hệ thống và sai phân được đánh giá thông qua thống kê Sargan/Hansen và thống kê Arellano-Bond. Các kiểm định Sargan/Hansen với giả thuyết null $\mathrm{H}_{0}$ : biến công cụ có tính ngoại sinh chặt chẽ, nghĩa là biến công cụ không tương quan với các sai số. Kiểm định Arellano-Bond được dùng để đánh giá tự tương quan chuỗi của các số dưới dạng sai phân bậc nhất. Vì thế, kết quả kiểm định tự tương quan chuỗi bậc nhất của các sai số bậc nhất $\mathrm{AR}(1)$ được bỏ qua trong khi tự tương quan chuỗi bậc hai của các sai số $\mathrm{AR}(2)$ được thực hiện dựa trên các sai phân bậc nhất của các sai số để dò tìm hiện tượng tự tương quan chuồi bậc nhất của các sai số.

\subsection{Dĩ liệu nghiên cứu}

Các biến là đầu tư công, sáu thành phần quản trị công, GDP bình quân đầu người thực, độ mở thương mại, lạm phát, và cơ sở hạ tầng. Dữ liệu được lấy từ World Development Indicators 
(WDI) và Worldwide Governance Indicators (WGI) của World Bank và Government Finance Statistics (GFS) của IMF. Mẫu nghiên cứu bao gồm 72 quốc gia đang phát triển gồm Albania, Angola, Argentina, Armenia, Azerbaijan, Bangladesh, Belize, Bhutan, Bosnia and Herzegovina, Botswana, Brazil, Bulgaria, Burkina Faso, Cabo Verde, Cambodia, Central African, China, Colombia, Congo Rep., Cote d'Ivoire, Croatia, Dominican Republic, Egypt, El Salvador, Equatorial Guinea, Gambia, Georgia, Ghana, Guatemala, Honduras, India, Indonesia, Iran, Jordan, Kazakhstan, Kuwait, Lao, Lebanon, Lesotho, Madagascar, Malawi, Malaysia, Maldives, Mali, Mauritius, Moldova, Mongolia, Morocco, Namibia, Nepal, Paraguay, Peru, Philippines, Romania, Russia, Saudi Arabia, Serbia, South Africa, Sri Lanka, St. Kitts and Nevis, St. Lucia, St. Vincent and the Grenadines, Tanzania, Thailand, Togo, Tunisia, Turkey, Ukraine, Uzbekistan, Việt Nam, Zambia, và Zimbabwe trong giai đoạn 2002 - 2018.

Thống kê mô tả các biến được trình bày trong Bảng 1 . Đặc biệt, các thành phần quản trị công có giá trị trung bình tương đối thấp với các giá trị âm. Điều này khá tương đồng với nghiên cứu của Li và Filer (2007) khi cho rằng các quốc gia đang phát triển có môi trường quản trị công kém (quản trị công dựa trên quan hệ). Bảng 2 trình bày ma trận tương quan giữa các biến với các hệ số tương quan có giá trị $<0.8$, giúp loại trừ khả năng cộng tuyến/đa cộng tuyến giữa các biến này. Trong khi đó, các biến thành phần quản trị công có hệ số tương quan với các giá trị tương đối cao, vượt qua 0.8 (Bảng 3). Do vậy, các biến thành phần này được sử dụng riêng lẻ trong các phương trình ước lượng để loại trừ khả năng cộng tuyến giữa các biến này.

\section{Bảng 1}

Thống kê mô tả các biến

\begin{tabular}{lccccc}
\hline \multicolumn{1}{c}{ Biến } & $\begin{array}{c}\text { Quan } \\
\text { sát }\end{array}$ & $\begin{array}{c}\text { Trung } \\
\text { bình }\end{array}$ & $\begin{array}{c}\text { Độ lệch } \\
\text { chuẩn }\end{array}$ & Nhỏ nhất & Lớn nhất \\
\hline Đầu tư công (GIN) & 1224 & 5.606 & 3.90 & 0.354 & 31.2714 \\
Thu nhập bình quân (GDP) & 1224 & 5263.07 & 5953.0 & 334.114 & 49578.5 \\
Độ mở thương mại (OPE) & 1224 & 82.544 & 33.574 & 22.105 & 210.374 \\
Lạm phát (INF) & 1224 & 6.20534 & 9.285 & -72.729 & 156.964 \\
Số thuê bao cố định trong 100 dân (TEL) & 1224 & 12.352 & 10.753 & 0.017 & 52.031 \\
\hline Chất lượng luật lệ (GO1) & 1224 & -0.422 & 0.591 & -1.826 & 1.646 \\
Nhà nước pháp quyền (GO2) & 1224 & -0.328 & 0.563 & -1.848 & 1.267 \\
Tiếng nói và giải trình (GO3) & 1224 & -0.302 & 0.754 & -2.699 & 1.364 \\
Kiểm soát tham nhũng (GO4) & 1224 & -0.279 & 0.578 & -2.236 & 1.240 \\
Hiệu quả chính phủ (GO5) & 1224 & -0.387 & 0.577 & -1.852 & 1.077 \\
Ổn định chính trị (GO6) & 1224 & -0.339 & 0.750 & -2.124 & 1.222 \\
\hline
\end{tabular}

Nguồn: Kết quả phân tích dữ liệu của nhóm nghiên cứu 


\section{Bảng 2}

Ma trận tương quan giữa các biến

\begin{tabular}{lccccc}
\hline & GIN & GDP & OPE & INF & TEL \\
\hline GIN & 1 & & & & \\
GDP & -0.033 & 1 & & & \\
OPE & $0.242^{* * *}$ & $0.156^{* * *}$ & 1 & 1 & \\
INF & -0.041 & $-0.085^{* * *}$ & $-0.067^{* *}$ & & \\
TEL & $-0.184^{* * *}$ & $0.728^{* * *}$ & $0.091^{* * *}$ & $-0.075^{* * *}$ & 1 \\
\hline
\end{tabular}

Ghi chú: ***, ** và * ký hiệu lần lượt cho các mức ý nghĩa $1 \%, 5 \%$, và $10 \%$

Nguồn: Kết quả phân tích dữ liệu của nhóm nghiên cứu

\section{Bảng 3}

Ma trận tương quan giữa các biến quản trị công

\begin{tabular}{lcccccc}
\hline & GO1 & GO2 & GO3 & GO4 & GO5 & GO6 \\
\hline GO1 & 1 & & & & & \\
GO2 & $0.801^{* * *}$ & 1 & & & & \\
GO3 & $0.553^{* * *}$ & $0.455^{* * *}$ & 1 & & & \\
GO4 & $0.681^{* * *}$ & $0.786^{* * *}$ & $0.407^{* * *}$ & 1 & & \\
GO5 & $0.886^{* * *}$ & $0.847^{* * *}$ & $0.586^{* * *}$ & $0.768^{* * *}$ & 1 & 1 \\
GO6 & $0.623^{* * *}$ & $0.499^{* * *}$ & $0.407^{* * *}$ & $0.615^{* * *}$ & $0.612^{* * *}$ & 1 \\
\hline
\end{tabular}

Ghi chú: ${ }^{* * *},{ }^{* *}$ và * ký hiệu lần lượt cho các mức ý nghĩa $1 \%, 5 \%$, và $10 \%$

Nguồn: Kết quả phân tích dữ liệu của nhóm nghiên cứu

\section{Kết quả và bàn luận}

\subsection{Uớc lựng S-GMM hai bước}

Các kết quả ước lượng được trình bày trong Bảng 4. Ở mỗi cột là một mô hình tương ứng với một thành phần quản trị công. Trong tất cả các quy trình ước lượng, chúng tôi dò ra được biến quản trị công là nội sinh, vì thế chúng tôi sử dụng biến quản trị công này như biến được công cụ trong thủ tục GMM (biến nội sinh) và các biến còn lại (đầu tư công, tăng trưởng kinh tế, độ mở thương mại, lạm phát, và cơ sở hạ tầng) như các biến công cụ trong thủ tục IV.

Các kết quả ở tất cả các mô hình đều cho thấy môi trường quản trị công làm giảm đầu tư công đều ở mức ý nghĩa $1 \%$. Tất cả các kết quả này đều nhất quán cao cho tất cả biến 6 thành phần quản trị công. Phát hiện này hoàn toàn tương đồng với nghiên cứu của Grigoli và Mills (2014), ủng hộ giả thuyết cho rằng các viên chức chính phủ sử dụng đầu tư công như một phương tiện để trục lợi. Ngoài ra, phát hiện này cũng tương đồng với kết quả nghiên cứu của Tanzi và Davoodi (1998) và Haque và Kneller (2015) khi cho rằng tham nhũng đưa đến gia tăng đầu tư công, và việc cải cách môi trường thể chế/quản trị công sẽ loại trừ dần tham nhũng và làm giảm đầu tư công. Điều này cho thấy dưới môi trường quản trị công kém, như ở các quốc gia đang phát triển trong nghiên cứu này ( $\mathrm{Li}$, Park, \& Li, 2004), việc thiết kế, ban hành và thực thi các chính sách liên quan đến các dự án đầu tư công không được giám sát công khai và minh bạch, tạo nên các lỗ hổng giúp cho các viên chức công và các nhóm lợi ích trục lợi từ các dự án công thông qua việc gia tăng đầu 
tư công. Việc cải thiện môi trường quản trị công vì thế sẽ loại trừ các lỗ hổng này và làm giảm đầu tư công. Do vậy, các phát hiện này nhấn mạnh rằng việc cải cách môi trường quản trị công ở các nước đang phát triển là một giải pháp cần thiết và quan trọng cho sự phát triển kinh tế. Li và cộng sự (2004) khẳng định quá trình cải thiện môi trường quản trị công sẽ chuyển đổi một quốc gia đang phát triển với môi trường quản trị công kém (quản trị công dựa trên quan hệ) thành một quốc gia phát triển với môi trường quản trị công tốt (quản trị dựa trên luật lệ) và tương ứng là sự thúc đẩy của môi trường quản trị công lên đầu tư công thay vì làm giảm. Phát hiện trong nghiên cứu này cũng hàm ý một giá trị ngưỡng của môi trường quản trị công, thể hiện sự khác biệt giữa các quốc gia đang phát triển và các quốc gia phát triển. Theo đó, các thành phần thể chế ở các quốc gia đang phát triển có giá trị nhỏ hơn zero (môi trường quản trị công kém) và do vậy quá trình cải cách và nâng cao môi trường thể chế sẽ giúp các quốc gia này một ngày nào đó trở thành các quốc gia phát triển với môi trường quản trị công tốt và khi đó môi trường quản trị công sẽ thúc đẩy thay vì làm giảm đầu tư công. Một điều có thể nhận thấy mà chính phủ ở các quốc gia đang phát triển có thể làm ngay để cải thiện môi trường quản trị công là nâng cao tính giải trình của các dự án đầu tư công bằng cách cho phép sự tham gia giám sát công khai và minh bạch của người dân (người thụ hưởng dự án) ở tất cả các khâu. Đặc biệt, một cơ chế kiểm soát tham nhũng hiệu quả cũng là một yêu cầu cần thiết ở các quốc gia này. Một số hình thức đầu tư công mới hiện nay ở các nước đang phát triển như hợp tác công tư (PPP), đấu thầu công khai, ... nên được đẩy mạnh áp dụng nhằm loại trừ tính trục lợi của các viên chức công và nhóm lợi ích.

Các kết quả ước lượng cũng chỉ ra tăng trưởng kinh tế thúc đẩy trong khi lạm phát và cơ sở hạ tầng làm giảm đầu tư công ở các quốc gia đang phát triển. Tác động dương của tăng trưởng kinh tế lên đầu tư công có thể được tìm thấy trong các nghiên cứu như Haque và Kneller (2015) và Gupta, Liu, và Mulas-Granados (2016) với lập luận rằng tăng trưởng kinh tế làm tăng nguồn thu thuế của chính phủ, do vậy giúp chính phủ có nguồn tiền để gia tăng chi đầu tư. Tương tự, Jin và Zou (2005) lập luận tác động tiềm tàng của lạm phát là làm tăng chi phí giao dịch và hạ thấp lợi nhuận, và do vậy sẽ làm giảm đầu tư. Điều này cũng đúng cho trường hợp đầu tư công như trong nghiên cứu này, đặc biệt việc gia tăng của lạm phát cũng khiến nhiều dự án công bị đình trệ vì làm tăng vốn đầu tư. Trong khi đó, sự phát triển cơ sở hạ tầng cũng làm giảm đầu tư công như trong nghiên cứu này chỉ ra. Điều này cho thấy khi cơ sở hạ tầng còn kém thì nhà nước sẽ gia tăng chi tiêu để hỗ trợ cho các hoạt động kinh tế phát triển, nhưng khi cơ sở hạ tầng tốt lên thì đầu tư công của nhà nước sẽ giảm dần để dành vốn cho các lĩnh vực khác.

\section{Bảng 4}

Quản trị công và đầu tư công: S-GMM hai bước, 2002 - 2018

Biến phụ thuộc: Đầu tư công $(\% \mathrm{GDP})$

\begin{tabular}{lcccccc}
\hline Biến & GO1 & GO2 & GO3 & GO4 & GO5 & GO6 \\
\hline Đầu tư công $(-1)$ & $0.935^{* * *}$ & $0.925^{* * *}$ & $0.944^{* * *}$ & $0.916^{* * *}$ & $0.936^{* * *}$ & $0.906^{* * *}$ \\
& $(0.015)$ & $(0.018)$ & $(0.025)$ & $(0.019)$ & $(0.015)$ & $(0.014)$ \\
Quản trị công & $-0.297^{* * *}$ & $-0.328^{* * *}$ & $-0.258^{* * *}$ & $-0.221^{* * *}$ & $-0.301^{* * *}$ & $-0.305^{* * *}$ \\
& $(0.073)$ & $(0.091)$ & $(0.084)$ & $(0.061)$ & $(0.072)$ & $(0.051)$ \\
Tăng trưởng kinh tế & $0.0006^{* * *}$ & $0.0006^{* * *}$ & $0.0006^{* *}$ & $0.0007^{* * *}$ & $0.0005^{* * *}$ & $0.0008^{* * *}$ \\
& $(0.0001)$ & $(0.0001)$ & $(0.0002)$ & $(0.0001)$ & $(0.0001)$ & $(0.0001)$ \\
Độ mở thương mại & 0.0006 & 0.001 & 0.001 & $0.002^{* *}$ & 0.001 & 0.001 \\
& $(0.001)$ & $(0.001)$ & $(0.001)$ & $(0.0001)$ & $(0.001)$ & $(0.001)$ \\
Lạm phát & $-0.005^{* * *}$ & $-0.005^{* *}$ & $-0.005^{* * *}$ & $-0.005^{* * *}$ & $-0.005^{* *}$ & $-0.004^{* * *}$ \\
& $(0.001)$ & $(0.002)$ & $(0.001)$ & $(0.001)$ & $(0.002)$ & $(0.001)$
\end{tabular}




\begin{tabular}{lcccccc}
\hline Biến & GO1 & GO2 & GO3 & GO4 & GO5 & GO6 \\
\hline Cơ sở hạ tầng & $-0.001^{* * *}$ & $-0.001^{* *}$ & $-0.001^{* *}$ & $-0.001^{* * *}$ & $-0.0006^{* *}$ & $-0.001^{* * *}$ \\
\hline Công cụ & $(0.0003)$ & $(0.0004)$ & $(0.000)$ & $(0.0004)$ & $(0.0003)$ & $(0.0002)$ \\
\hline Quốc gia/Quan sát & 37 & 37 & 37 & 36 & 37 & 36 \\
\hline AR(2) test & $72 / 1008$ & $72 / 1008$ & $72 / 1008$ & $72 / 936$ & $72 / 1008$ & $72 / 1008$ \\
\hline Sargan test & 0.602 & 0.601 & 0.601 & 0.609 & 0.610 & 0.599 \\
\hline Hansen test & 0.117 & 0.390 & 0.186 & 0.125 & 0.239 & 0.238 \\
\hline
\end{tabular}

Ghi chú: ${ }^{* * *},{ }^{* *}$ và * ký hiệu lần lượt cho các mức ý nghĩa $1 \%, 5 \%$, và $10 \%$

Nguồn: Kết quả phân tích dữ liệu của nhóm nghiên cứu

\subsection{Kiểm tra tính bền}

Để kiểm tra tính bền, chúng tôi sử dụng S-GMM một bước để ước lượng lại phương trình (1). Các kết quả tương ứng cho tất cả các mô hình được thể hiện trong Bảng 5 . Nhất quán với các ước lượng $\mathrm{S}-\mathrm{GMM}$ hai bước trong Bảng 4 , chúng tôi tìm thấy môi trường quản trị công làm giảm đầu tư công. Tăng trưởng kinh tế làm tăng trong khi lạm phát và cơ sở hạ tầng làm giảm đầu tư công mặc dù không có ý nghĩa thống kê. Ngoài ra, độ mở thương mại cũng là một yếu tố tác động dương có ý nghĩa lên đầu tư công. Các phát hiện này được khẳng định bằng các kiểm định được chỉ ra ở bên dưới Bảng 5 , kiểm định Sargan tests và kiểm định Arellano-Bond $\mathrm{AR}(2)$, hàm ý rằng các ước lượng S-GMM một bước đáng tin cậy.

\section{Bảng 5}

Quản trị công và đầu tư công: S-GMM một bước, 2002 - 2018

Biến phụ thuộc: Đầu tư công (\%GDP)

\begin{tabular}{lcccccc}
\hline Biến & GO1 & GO2 & GO3 & GO4 & GO5 & GO6 \\
\hline Đầu tư công (-1) & $0.917^{* * * *}$ & $0.926^{* * *}$ & $0.934^{* * *}$ & $0.907^{* * *}$ & $0.910^{* * *}$ & $0.898^{* * *}$ \\
& $(0.029)$ & $(0.029)$ & $(0.030)$ & $(0.027)$ & $(0.028)$ & $(0.025)$ \\
Quản trị công & $-0.525^{* *}$ & $-0.592^{* * *}$ & $-0.509^{* *}$ & $-0.372^{*}$ & $-0.523^{* * *}$ & $-0.340^{* *}$ \\
& $(0.209)$ & $(0.229)$ & $(0.251)$ & $(.206)$ & $(0.182)$ & $(0.135)$ \\
Tăng trưởng kinh tế & 0.0001 & 0.0000 & 0.0002 & 0.0005 & 0.0003 & $0.0006^{* *}$ \\
& $(0.0004)$ & $(0.0004)$ & $(0.0005)$ & $(0.0003)$ & $(0.0003)$ & $(0.0003)$ \\
Độ mở thương mại & $0.004^{* *}$ & $0.003^{*}$ & $0.004^{*}$ & $0.004^{*}$ & $0.004^{* *}$ & 0.003 \\
& $(0.002)$ & $(0.002)$ & $(0.002)$ & $(0.002)$ & $(0.002)$ & $(0.002)$ \\
Lạm phát & -0.004 & -0.005 & -0.005 & -0.005 & -0.005 & -0.002 \\
& $(0.009)$ & $(0.009)$ & $(0.009)$ & $(0.009)$ & $(0.009)$ & $(0.008)$ \\
Cơ sở hạ tầng & -0.0004 & 0.0002 & -0.001 & -0.0008 & -0.0007 & $-0.001^{* *}$ \\
& $(0.0007)$ & $(0.0009)$ & $(0.0008)$ & $(0.0007)$ & $(0.0007)$ & $(0.0005)$ \\
\hline Công cụ & 34 & 35 & 36 & 35 & 35 & 36 \\
\hline Quốc gia/Quan sát & $72 / 936$ & $72 / 936$ & $72 / 1008$ & $72 / 936$ & $72 / 936$ & $72 / 1008$ \\
\hline AR(2) test & 0.504 & 0.499 & 0.490 & 0.506 & 0.522 & 0.492 \\
\hline Sargan test & 0.136 & 0.445 & 0.141 & 0.104 & 0.102 & 0.238 \\
\hline
\end{tabular}

Ghi chú: ${ }^{* * *},{ }^{* *}$ và * ký hiệu lần lượt cho các mức ý nghĩa $1 \%, 5 \%$, và $10 \%$

Nguồn: Kết quả phân tích dữ liệu của nhóm nghiên cứu 


\section{Kết luận và hàm ý chính sách}

Xuất phát từ thực tiễn là môi trường quản trị công/thể chế có tác động nhất định lên đầu tư công, bài viết đánh giá thực nghiệm tác động của môi trường quản trị công lên đầu tư công cho dữ liệu bảng cân bằng của 72 quốc gia đang phát triển trong giai đoạn $2002-2018$ bằng phương pháp ước lượng GMM Arellano-Bond hệ thống một bước và hai bước. Kết quả cho thấy môi trường quản trị công làm giảm đầu tư công ở các quốc gia đang phát triển, ủng hộ giả thuyết là các viên chức chính phủ sử dụng đầu tư công như một phương tiện để trục lợi. Ngoài ra, tăng trưởng kinh tế, lạm phát, và cơ sở hạ tầng cũng là các yếu tố quyết định có ý nghĩa của đầu tư công ở các quốc gia này.

Các phát hiện trong nghiên cứu này đòi hỏi sự thận trọng trong quá trình thiết kế, ban hành và thực thi các chính sách đầu tư công và các dự án công. Hàm ý là chính phủ các quốc gia đang phát triển, trong đó có chính phủ Việt Nam, cần cải cách mạnh mẽ môi trường quản trị công để tạo các điều kiện thuận lợi cho việc thúc đẩy đầu tư công trong tương lai. Đặc biệt, quá trình cải thiện môi trường quản trị công ở các quốc gia đang phát triển sẽ giúp loại trừ các lỗ hổng mà các viên chức công và các nhóm lợi ích lợi dụng để trục lợi trong các dự án công. Quá trình cải thiện và cải cách môi trường quản trị công liên tục sẽ giúp các quốc gia này trong tương lai sẽ chuyển đổi từ môi trường thể chế kém (quản trị công dựa trên quan hệ) sang môi trường thể chế tốt (quản trị công dựa trên luật lệ) và khi đó môi trường quản trị công sẽ thúc đẩy thay vì làm giảm đầu tư công ở các quốc gia này.

\section{LÒ̀I CẢM ƠN}

Nghiên cứu này được thực hiện dựa trên tài trợ kinh phí của Trường Đại học Tài chính Marketing (UFM).

\section{Tài liệu tham khảo}

Acemoglu, D., Johnson, S., \& Robinson, J. A. (2005). Institutions as a fundamental cause of longrun growth. Handbook of Economic Growth, 1(A), 385-472.

Agyei, S. K. (2017). Explaining public investment dynamics in Sub-Saharan Africa: The role of country governance structures. Cogent Economics \& Finance, 5(1), Article 1323987. doi:10.1080/23322039.2017.1323987

Arellano, M., \& Bond, S. (1991). Some tests of specification for panel data: Monte Carlo evidence and an application to employment equations. The Review of Economic Studies Journal, 58(2), 277-297.

Arellano, M., \& Bover, O. (1995). Another look at the instrumental variable estimation of errorcomponents models. Journal of Econometrics, 68(1), 29-51.

Baum, C. F., Schaffer, M. E., \& Stillman, S. (2003). XTIVREG2: Stata module to perform extended IV/2SLS, GMM AND AC/HAC, LIML and k-class regression for panel data models. The Stata Journal, 3.

De la Croix, D., \& Delavallade, C. (2009). Growth, public investment and corruption with failing institutions. Economics of Governance, 10(3), 187-219.

Delavallade, C. (2006). Corruption and distribution of public spending in developing countries. Journal of Economics and Finance, 30(2), 222-239. 
Duchin, R., \& Sosyura, D. (2012). The politics of government investment. Journal of Financial Economics, 106(1), 24-48.

Grigoli, F., \& Mills, Z. (2014). Institutions and public investment: An empirical analysis. Economics of Governance, 15(2), 131-153.

Gupta, S., Liu, E. X., \& Mulas-Granados, C. (2016). Now or later? The political economy of public investment in democracies. European Journal of Political Economy, 45, 101-114.

Gwatipedza, J., \& Janus, T. (2019). Public investment under autocracy and social unrest. Economics \& Politics, 31(1), 112-135.

Haque, M. E., \& Kneller, R. (2015). Why does public investment fail to raise economic growth? The role of corruption. The Manchester School, 83(6), 623-651.

Haughwout, A. F. (2002). Public infrastructure investments, productivity and welfare in fixed geographic areas. Journal of Public Economics, 83(3), 405-428.

Holtz-Eakin, D., Newey, W., \& Rosen, H. S. (1988). Estimating vector autoregressions with panel data. Econometrica: Journal of the Econometric Society, 56(6), 1371-1395.

Hope, K. R. (2009). Capacity development for good governance in developing countries: Some lessons from the field. International Journal of Public Administration, 3(2), 728-740.

Jin, J., \& Zou, H. F. (2005). Fiscal decentralization, revenue and expenditure assignments, and growth in China. Journal of Asian Economics, 16(6), 1047-1064.

Judson, R. A., \& Owen, A. L. (1999). Estimating dynamic panel data models: A guide for macroeconomists. Economics Letters, 65(1), 9-15.

Kaufmann, D., Kraay, A., \& Mastruzzi, M. (2011). The worldwide governance indicators: Methodology and analytical issues. Hague Journal on the Rule of Law, 3(2), 220-246.

Li, S., \& Filer, L. (2007). The effects of the governance environment on the choice of investment mode and the strategic implications. Journal of World Business, 42(1), 80-98.

Li, S., Park, S. H., \& Li, S. (2004). The great leap forward: The transition from relation-based governance to rule-based governance. Organizational Dynamics, 33(1), 63-78.

North, D. (1990). Institutions, institutional change and economic performance (Political economy of institutions and decisions). Cambridge, UK: Cambridge University Press.

Roodman, D. (2009). How to do xtabond2: An introduction to difference and system GMM in Stata. The Stata Journal, 9(1), 86-136.

Tanzi, V., \& Davoodi, H. (1998). Corruption, public investment, and growth. In The welfare state, public investment, and growth (pp. 41-60). Tokyo, Japan: Springer.

World Bank. (2017). World development report 2017: Governance and the law. Washington, DC: World Bank.

Xu, Y., \& Yao, Y. (2015). Informal institutions, collective action, and public investment in rural China. American Political Science Review, 109(2), 371-391. 